DXA and $3(1.3 \%)$ had indication for treatment by FRAX without DXA but they lost it by FRAX with DXA. We found a moderate level of agreement in the indication for treatment between FRAX with and without DXA (kappa=0.595; $p<0.001$ ). The use of DXA in FRAX estimated a significant higher median FR, both for major osteoporotic fracture $(2.4 \%[0.8-31.0]$ vs $1.8 \%$ [0.6-20.0]; $p<0.001)$ and for hip fracture $(0.5 \%[0.0-23.0]$ vs $0.2 \%$ [0.0-14.0]; $p<0.001)$.

We found significant correlations between FR and some disease-related variables (table 1).

Table 1. Correlations between the risk of fracture estimated by FRAX and disease-related variables.

\begin{tabular}{|c|c|c|c|c|c|c|c|}
\hline & & & $\begin{array}{l}\text { Disease } \\
\text { duration }\end{array}$ & BASDAI & ASDAS-CRP & BASMI & BASFI \\
\hline \multirow{8}{*}{ 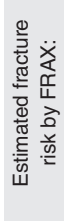 } & \multirow{4}{*}{$\begin{array}{c}\text { without } \\
\text { DXA }\end{array}$} & \multirow{3}{*}{$\begin{array}{l}\text { major osteoporotic } \\
\text { fracture } \\
\text { hip fracture }\end{array}$} & $r=0.352$ & $r=0.204$ & $r=0.214$ & $r=0.301$ & $r=0.317$ \\
\hline & & & $p<0.001$ & $p=0.002$ & $p=0.001$ & $p<0.001$ & $p<0.001$ \\
\hline & & & $r=0.389$ & $r=0.142$ & $r=0.170$ & $r=0.305$ & $r=0.275$ \\
\hline & & & $p<0.001$ & $p=0.034$ & $p=0.011$ & $p<0.001$ & $p<0.001$ \\
\hline & & major osteoporotic & $r=0.227$ & $r=0.314$ & $r=0.356$ & $r=0.293$ & $r=0.379$ \\
\hline & with & fracture & $p=0.014$ & $p=0.001$ & $p<0.001$ & $p=0.002$ & $p<0.001$ \\
\hline & DXA & hip fracture & n.s. & $r=0.197$ & $r=0.269$ & $r=0.271$ & $r=0.258$ \\
\hline & & & & $p=0.036$ & $p=0.004$ & $p=0.004$ & $p=0.006$ \\
\hline
\end{tabular}

Conclusion: Our results showed that a similar number of patients had indication for pharmacological treatment by FRAX both with and without DXA. Although the inclusion of DXA resulted in a higher estimated FR by FRAX, the observed moderate level of agreement between FRAX with and without DXA suggests that the FR estimation by FRAX, even without DXA, may be a reasonable approach in SpA patients. In line with literature, we found significant associations between the estimated risk fracture by FRAX and some disease activity and function measures.

Disclosure of Interests: Bruno Miguel Fernandes: None declared, Salomé Garcia: None declared, Sara Ganhão: None declared, Maria Rato: None declared, Filipe Pinheiro: None declared, Miguel Bernardes Speakers bureau: Abbvie, Amgen, Biogen, Eli-Lilly, Glaxo-Smith-Kline, Pfizer, Janssen, Novartis, Lúcia Costa: None declared

DOI: 10.1136/annrheumdis-2020-eular.1570

\section{SAT0450 GLUCOCORTICOID-INDUCED OSTEOPOROSIS IN PATIENTS WITH CHRONIC INFLAMMATORY RHEUMATIC DISEASES: A MULTIVARIATE LINEAR REGRESSION ANALYSIS IDENTIFYING PREDICTIVE FACTORS FOR LOW BONE MASS}

E. Wiebe ${ }^{1}$, D. Freier ${ }^{1}$, D. Huscher ${ }^{2,3}$, R. Biesen ${ }^{1}$, S. Hermann ${ }^{1}$, F. Buttgereit ${ }^{1}$. ${ }^{1}$ Charité University Medicine Berlin, Rheumatology and Clinical Immunology, Berlin, Germany; ${ }^{2}$ Charité University Medicine Berlin, Biometry and Clinical Epidemiology, Berlin, Germany; ${ }^{3}$ Charité University Medicine Berlin, Berlin Institute of Health, Berlin, Germany

Background: Rheumatic diseases are associated with increased systemic bone loss and fracture risk related to chronic inflammation, disease-specific, general and demographic risk factors as well as treatment with glucocorticoids (GC). Yet, there is evidence that GCs may, by adequately suppressing systemic inflammation, also have a positive effect on bone mineral density (BMD) and fracture risk ${ }^{1}$. Objectives: The purpose of this study was to investigate the prevalence of osteoporosis and fragility fractures in patients with inflammatory rheumatic diseases and to analyze the impact that treatment with GCs, other known risk factors and preventive measures have on bone health in these patients.

Methods: Rh-GIOP is an ongoing prospective observational study collecting and analyzing disease- and bone-related data from patients with chronic inflammatory rheumatic diseases and psoriasis treated with GCs. In this cross-sectional analysis, we evaluated the initial visit of 1091 patients. A multivariate linear regression model with known or potentially influential factors adjusted for age and sex was used to identify predictors of BMD as measured by dual-energy X-ray absorptiometry (DXA). Multiple imputation was applied for missing baseline covariate data.

Results: In the total cohort of 1091 patients $(75 \%$ female of which $87.5 \%$ were postmenopausal) with a mean age of $62.1( \pm 13.2)$ years, the prevalence of osteoporosis by DXA was $21.7 \%$, while fragility fractures have occurred in $31.2 \%$ of the study population (6.7\% vertebral, $27.7 \%$ non-vertebral). Current GC therapy was common $(64.9 \%)$, with a median daily dose of $5.0 \mathrm{mg}[0.0 ; 7.5]$, a mean life-time total GC dose of $17.7 \mathrm{~g}( \pm 24.6)$, and a mean GC therapy duration of 7.8 years $( \pm 8.5)$. Bisphosphonates were the most commonly used anti-osteoporotic drug $(12.6 \%)$.

Multivariate analysis showed that BMD as expressed by the minimum T-Score at all measured sites was negatively associated with higher age, female sex and menopause as well as Denosumab and Bisphosphonate treatment. A positive association with BMD was found for body mass index as well as current and life-time (cumulative) GC dose. While comedication with proton-pump-inhibitors significantly predicted low bone mass, concomitant use of non-steroidal anti-inflammatory drugs showed a positive association with BMD. Of the measured bone-specific laboratory parameters, higher alkaline phosphatase levels were determinants of low DXA-values, while the association was positive for gamma-glutamyltransferase.

BMD was neither predicted by duration of GC treatment nor by treatment with disease modifying anti-rheumatic drugs.

Predictive variables for BMD differed at the respective anatomical site. While treatment with Denosumab predicted low bone mass at the lumbar spine and not at the femoral neck, the opposite was true for health assessment questionnaire (HAQ) score. Current and life-time GC-dose as well as direct sun-exposure of more than 30 minutes daily were positively associated with bone mass at the femoral sites only. Conclusion: This cross-sectional analysis of a prospective cohort study quantified the prevalence of osteoporosis and identified predictive variables of BMD in patients with rheumatic diseases.

Multivariate analyses corroborated low BMD to be predicted by traditional factors like age, female sex and menopause but showed current and well as life-time GC dose to be positively associated with BMD in our cohort of patients with chronic inflammatory rheumatic diseases. This suggests that optimal management of disease activity with GCs might be beneficial in order to avoid bone loss due to inflammation.

\section{References:}

[1] Güler-Yüksel et al. "Glucocorticoids, Inflammation and Bone." Calcified Tissue International (January 08 2018).

Disclosure of Interests: Edgar Wiebe: None declared, Desiree Freier: None declared, Dörte Huscher: None declared, Robert Biesen: None declared, Sandra Hermann: None declared, Frank Buttgereit Grant/research support from: Amgen BMS, Celgene, Generic Assays, GSK, Hexal, Horizon, Lilly, medac, Mundipharma, Novartis, Pfizer, Roche, and Sanofi. DOI: 10.1136/annrheumdis-2020-eular.4427

\section{SAT0451 COMPARISON OF PHARMACOKINETIC, PHARMACODYNAMIC AND SAFETY OF A TERIPARATIDE BIOSIMILAR AND REFERENCE TERIPARATIDE}

I. Singh ${ }^{1}$, A. Attrey ${ }^{2}$, R. Patel ${ }^{2}$, S. Khambhampaty ${ }^{1}$, V. Jose ${ }^{1} .{ }^{1}$ Intas

Pharmaceuticals Ltd, Matoda, Ahmedabad, India; ${ }^{2}$ Lambda Therapeutic

Research Ltd., Ahmedabad, India

Background: Intas is developing a biosimilar teriparatide. This teriparatide biosimilar has shown similarity to European Union approved teriparatide (EU teriparatide) and United States approved teriparatide (US-teriparatide) in analytical (structural and functional assays) and animal studies ${ }^{1}$.

Objectives: To primarily asses pharmacokinetic (PK) equivalence and to compare pharmacodynamic (PD) and safety profiles of teriparatide biosimilar against EU- and US-teriparatide in healthy men and postmenopausal women with potentially similar in-clinic real-life user profiles.

Methods: In this assessor-blind, three-period study, 105 subjects were randomized (1:1:1) to receive single subcutaneous $20 \mu \mathrm{g}$ dose each of teriparatide biosimilar, EU-teriparatide, and US-teriparatide in a crossover manner on 3 consecutive days. The primary PK endpoints were area under the curve (AUC) from time zero to $t\left(A \cup C_{0-1}\right), A \cup C$ from time zero to infinity $\left(A \cup C_{0-\infty}\right)$, and maximum concentration $\left(\mathrm{C}_{\max }\right)$. Secondary endpoints were total serum calcium level as a pharmacodynamic marker and safety.

Results: The mean age of 48 men was 38.4 years and 57 women was 43.9 years. Mean serum teriparatide concentrations were similar for teriparatide biosimilar, EU- and US-teriparatide (Figure 1). The $90 \%$ confidence intervals (Cl) of the geometric least square mean ratios (GMR) of $C_{\text {max }}, A C_{0-t}$ and $A C_{0-\infty}$ of teriparatide biosimilar relative to EU- and US-teriparatide were within the predefined acceptance range of $80.00 \%$ to $125.00 \%$ (Table 1). The baseline-adjusted and non-adjusted corrected total serum calcium levels were similar (Table 2). A total of 42 adverse events (AE) were reported by 31 (29.52\%) subjects ( 9 subjects, teriparatide biosimilar; 14 subjects, EU-teriparatide; 13 subjects, US-teriparatide), with headache and pain in extremity being the most common AEs. No death or serious AEs were reported. 